\title{
ON THE GLOBAL SOLVABILITY OF SOLUTIONS \\ TO A QUASILINEAR WAVE EQUATION WITH \\ LOCALIZED DAMPING AND SOURCE TERMS
}

E. CABANILLAS LAPA, Z. HUARINGA SEGURA, AND F. LEON BARBOZA

Received 18 July 2004 and in revised form 9 March 2005

We prove existence and uniform stability of strong solutions to a quasilinear wave equation with a locally distributed nonlinear dissipation with source term of power nonlinearity of the type $u^{\prime \prime}-M\left(\int_{\Omega}|\nabla u|^{2} d x\right) \Delta u+a(x) g\left(u^{\prime}\right)+f(u)=0$, in $\left.\Omega \times\right] 0,+\infty[, u=0$, on $\Gamma \times] 0,+\infty\left[, u(x, 0)=u_{0}(x), u^{\prime}(x, 0)=u_{1}(x)\right.$, in $\Omega$.

\section{Introduction}

Let $\Omega$ be a bounded domain of $\mathbb{R}^{N}$ with a smooth boundary $\Gamma=\partial \Omega$. We consider the initial-boundary value problem

$$
\begin{gathered}
\left.u^{\prime \prime}-M\left(\int_{\Omega}|\nabla u|^{2} d x\right) \Delta u+a(x) g\left(u^{\prime}\right)+f(u)=0, \quad \text { in } \Omega \times\right] 0,+\infty[, \\
u=0, \quad \text { on } \Gamma \times] 0,+\infty[, \\
u(x, 0)=u_{0}(x), \quad u^{\prime}(x, 0)=u_{1}(x), \quad \text { in } \Omega,
\end{gathered}
$$

where $M(s)$ is a $C^{1}$-class function on $\left[0,+\infty\right.$ [ satisfying $M(s) \geq m_{0}>0$, for $s \geq 0$, with $m_{0}$ constant, $a$ is a smooth nonnegative function but vanishes somewhere in $\bar{\Omega}, f(u)$ is a nonlinear term like $f(u) \sim-|u|^{\alpha} u$, and $g$ is a real-valued function.

The problem (1.1), when $M(s)=1$ and $f$ is some type of nonlinear function, has been studied by Zuazua [10] and Nakao [9]. Recently, Cabanillas et al. have treated in [2, 3] a more delicate case where $M$ is not a constant function $\left(f(u)=0,-h_{0} u\right)$. KouemouPatcheu [6] investigated the case $M(s)=a_{0}+b s$ with $a(x)=1$ in $\Omega$ and $f(u)=0$. We fix $x^{0} \in \mathbb{R}^{N}$ and we set

$$
m(x)=x-x^{0}, \quad R=\sup \{|m(x)| ; x \in \Omega\}, \quad \Gamma_{0}=\{x \in \Gamma ; m(x) \cdot \nu(x)>0\},
$$

where $\nu(x)$ denotes the outward unit normal at $x \in \Gamma$. Let $a=a(x)$ be a smooth nonnegative function such that

$$
a(x) \geq a_{0}>0, \quad \text { a.e. in } \omega,
$$


where $\omega$ is a neighborhood of $\Gamma_{0}$ and $a_{0}$ is a positive constant. By neighborhood of $\Gamma_{0}$, we actually mean the intersection of $\Omega$ and a neighborhood of $\Gamma_{0}$.

The goal of this work is to obtain global existence and decay estimates of the strong solutions of the quasilinear wave equation (1.1) when $M$ is not a constant function, the function $a$ satisfies (1.3), $g$ is a $C^{1}$, odd, increasing function, and $f(u) \sim-|u|^{\alpha} u$.

\section{Preliminaries and main result}

Throughout this paper, the functions considered are all real valued and the notations for their norm are adopted as usual (e.g., Lions [7]).

We consider the following general hypotheses.

(A.1) Assumptions on $M$ :

$$
\begin{gathered}
M \in C^{1}\left(\left[0,+\infty[), \quad M(s) \geq m_{0}>0, \quad \forall s \geq 0,\right.\right. \\
\left|M^{\prime}(s)\right| \leq \beta s^{\gamma / 2}, \quad \forall s \geq 0
\end{gathered}
$$

for some constants $\beta \geq 0, \gamma \geq 0$.

(A.2) Assumptions on $a$ :

$$
a \in C^{2}(\Omega) \cap C(\bar{\Omega}), \quad|\Delta a(x)| \leq a_{1} a(x), \quad a_{1}>0 .
$$

(A.3) Assumptions on $f$ :

$f$ is a $C^{1}$-class function on $\mathbb{R}$ and satisfies

$$
|f(u)| \leq h_{0}|u|^{\alpha+1}, \quad\left|f^{\prime}(u)\right| \leq h_{0}|u|^{\alpha}, \quad \forall u \in \mathbb{R},
$$

with some constant $h_{0}>0$ and

$$
0<\alpha<\frac{2}{(N-4)^{+}}
$$

where $(N-4)^{+}=\max \{N-4,0\}$.

(A.4) $g$ is a $C^{1}$ odd increasing function and

$$
\begin{gathered}
C_{1}|s| \leq|g(s)| \leq C_{2}|s|^{q} \quad \text { if }|s| \geq 1 \text { with } 1 \leq q \leq \frac{2}{(N-4)^{+}} \\
C_{3}|s|^{p+1} \leq g(s) s \quad \text { if }|s|<1,1 \leq p<+\infty
\end{gathered}
$$

where $C_{i}, i=1,2,3$, are positive constants.

We have the following fundamental inequalities.

Lemma 2.1 (Sobolev-Poincaré inequality). Let $\alpha$ be a number with $0 \leq \alpha<\infty(N=1,2)$ or $0 \leq \alpha \leq 4 /(N-2)(N \geq 3)$, then there is a constant $C_{*}>0$ such that

$$
|u|_{\alpha+2} \leq C_{*}|\nabla u|_{2} \quad \text { for } u \in H_{0}^{1}(\Omega) .
$$

Lemma 2.2 (Gagliardo-Nirenberg inequality). Let $1 \leq r<q \leq+\infty$ and $p \leq q$. Then, the inequality

$$
|u|_{W^{k, q}} \leq C|u|_{W^{m, p}}^{\theta}|u|_{r}^{1-\theta} \quad \text { for } u \in W^{m, p} \cap L^{r}
$$


holds with some $C>0$ and

$$
\theta=\left(\frac{k}{N}+\frac{1}{r}-\frac{1}{q}\right)\left(\frac{m}{N}+\frac{1}{r}-\frac{1}{p}\right)^{-1}
$$

provided that $0<\theta \leq 1$ (assume that $0<\theta<1$ if $q=+\infty$ ).

LEMMA 2.3. Let $E:[0,+\infty[\rightarrow[0,+\infty[$ be a nonincreasing function and assume that there are two constants $p \geq 1$ and $A>0$ such that

$$
\int_{S}^{+\infty} E^{(p+1) / 2}(t) d t \leq A E(S), \quad 0 \leq S<+\infty
$$

Then,

$$
E(t) \leq \begin{cases}C E(0) e^{-\lambda t}, & \forall t \geq 0 \text { if } p=1 \\ C E(0)(1+t)^{-2 /(p-1)}, & \forall t \geq 0 \text { if } p>1\end{cases}
$$

where $C$ and $\lambda$ are positive constants independent of the initial energy $E(0)$.

We will construct a stable set in $H_{0}^{1} \cap H^{2}$. For this, we define the functionals

$$
\begin{aligned}
J(u) & =\frac{1}{2} \widetilde{M}\left(|\nabla u|^{2}\right)+\int_{\Omega} F(u) d x, \quad \text { for } u \in H_{0}^{1}, \\
I(u) & =M\left(|\nabla u|^{2}\right)|\nabla u|^{2}+\int_{\Omega} f(u) \cdot u d x, \quad \text { for } u \in H_{0}^{1}, \\
E(u, v) & =\frac{1}{2}|v|^{2}+J(u), \quad \text { for }(u, v) \in H_{0}^{1} \times L^{2},
\end{aligned}
$$

where

$$
\widetilde{M}(s)=\int_{0}^{s} M(\xi) d \xi, \quad F(\lambda)=\int_{0}^{\lambda} f(s) d s .
$$

Lemma 2.4. Let $0<\alpha<4 /(N-4)^{+}$. Then, for any $K>0$, there exists a number $\varepsilon_{0}=\varepsilon_{0}(K)$ such that if $|\Delta u| \leq K$ and $|\nabla u| \leq \varepsilon_{0}$,

$$
J(u) \geq \frac{m_{0}}{4}|\nabla u|^{2}, \quad I(u) \geq \frac{m_{0}}{2}|\nabla u|^{2} .
$$

Proof. By the Gagliardo-Nirenberg inequality, we deduce that

$$
|u|_{\alpha+2}^{\alpha+2} \leq C|u|_{2 N /(N-2)}^{(\alpha+2)(1-\theta)}|\Delta u|^{(\alpha+2) \theta} \leq C|\nabla u|^{(\alpha+2)(1-\theta)}|\Delta u|^{(\alpha+2) \theta}
$$


222 Equation with localized damping and source terms

with

$$
\theta=\left(\frac{N-2}{2 N}-\frac{1}{\alpha+2}\right)^{+}\left(\frac{2}{N}+\frac{N-2}{2 N}-\frac{1}{2}\right)^{-1}=\left(\frac{(N-2) \alpha-4}{2(\alpha+2)}\right)^{+} \leq 1
$$

Here, we note that

$$
(\alpha+2)(1-\theta)-2= \begin{cases}\alpha & \text { if } 0<\alpha<\frac{4}{N-2} \\ & (0<\alpha<+\infty, \text { for } N=1,2), \\ \frac{(4-N) \alpha+4}{2} & \text { if } \frac{4}{N-2}<\alpha<\frac{4}{N-4} \\ & \left(\frac{4}{N-2}<\alpha<+\infty, N=3,4\right) .\end{cases}
$$

Hence, if $|\Delta u| \leq K$, we get

$$
\begin{aligned}
J(u) & \geq \frac{m_{0}}{2}|\nabla u|^{2}-\frac{h_{0}}{\alpha+2}|u|_{\alpha+2}^{\alpha+2} \\
& \geq \frac{m_{0}}{2}|\nabla u|^{2}-C h_{0}|\nabla u|^{(\alpha+2)(1-\theta)}|\Delta u|^{(\alpha+2) \theta} \\
& \geq\left\{\frac{m_{0}}{2}-C h_{0} K^{(\alpha+2) \theta}|\nabla u|^{(\alpha+2)(1-\theta)-2}\right\}|\nabla u|^{2} .
\end{aligned}
$$

Using (2.17), we can define $\varepsilon_{0}=\varepsilon_{0}(K)$ by

$$
C K^{(\alpha+2) \theta} \varepsilon_{0}^{(\alpha+2)(1-\theta)-2} \leq \frac{m_{0}}{4}
$$

Thus, we obtain

$$
J(u) \geq \frac{m_{0}}{4}|\nabla u|^{2}
$$

if $|\nabla u| \leq \varepsilon_{0}$. In a completely analogous way, we can get (2.14) for $I(u)$.

We define our stable set $W_{K}$ by

$$
W_{K}=\left\{(u, v) \in\left(H_{0}^{1} \cap H^{2}\right) \times H_{0}^{1}:|\Delta u|<K,|\nabla v|<K, \sqrt{4 m_{0}^{-1} E\left(u_{0}, v_{0}\right)}<\varepsilon_{0}\right\}
$$

for $K>0$.

Remark 2.5. If we consider $f(u) \cdot u \geq 0$, then we need not take $\varepsilon_{0}(K)$, and $W_{K}$ is replaced by

$$
\widetilde{W}_{K}=\left\{(u, v) \in\left(H_{0}^{1} \cap H^{2}\right) \times H_{0}^{1}:|\Delta u|<K,|\nabla v|<K\right\} .
$$




\section{Statement of the results}

In this section, we will state our main theorem.

Theorem 3.1 (local existence). Let initial data $\left\{u_{0}, u_{1}\right\}$ belong to $\left(H_{0}^{1} \cap H^{2}\right) \times H_{0}^{1}$ and let the assumptions (A.1)-(A.4) be fulfilled. Then there exists a unique local solution $u$ of (1.1) belonging to

$$
C_{w}^{0}\left(\left[0, T\left[; H_{0}^{1} \cap H^{2}\right) \cap C_{w}^{1}\left(\left[0, T\left[; H_{0}^{1}\right) \cap C^{0}\left(\left[0, T\left[; H_{0}^{1}\right) \cap C^{1}\left(\left[0, T\left[; L^{2}(\Omega)\right)\right.\right.\right.\right.\right.\right.\right.\right.
$$

for some $T=T\left(\left|\Delta u_{0}\right|,\left|\nabla u_{1}\right|\right)>0$.

Moreover, at least one of the following statements is valid:

(i) $T=+\infty$,

(ii) $\left|\nabla u^{\prime}(t)\right|^{2}+|\Delta u(t)|^{2} \rightarrow \infty$ as $t \rightarrow T^{-}$,

(iii) $M\left(|\nabla u(t)|^{2}\right) \rightarrow 0$ as $t \rightarrow T^{-}$.

The proof of this theorem is well known.

Theorem 3.2 (global existence and decay property). Suppose (A.1)-(A.4) hold. Then there exists an open set $S_{0}$ in $\left(H_{0}^{1} \cap H^{2}\right) \times H_{0}^{1}$, which contains $(0,0)$ such that if $\left(u_{0}, u_{1}\right) \in S_{0}$, the problem (1.1) admits a unique global solution $u(t)$ on the class

$$
L^{\infty}\left(\left[0,+\infty\left[; H_{0}^{1} \cap H^{2}\right) \cap W^{1, \infty}\left(\left[0,+\infty\left[; H_{0}^{1}\right) \cap W^{2, \infty}\left(\left[0,+\infty\left[; L^{2}\right) .\right.\right.\right.\right.\right.\right.
$$

Moreover, the energy determined by the solution $u$ has the decay states

$$
\begin{gathered}
E\left(u(t), u^{\prime}(t)\right) \leq C_{0} e^{-\lambda t} \quad \text { if } p=1, \\
E\left(u(t), u^{\prime}(t)\right) \leq \widetilde{C}_{0}(1+t)^{-2 /(p-1)} \quad \text { if } p>1,
\end{gathered}
$$

where $C_{0}, \widetilde{C}_{0}$, and $\lambda$ are certain positive constants depending on $\left|\nabla u_{0}\right|,\left|u_{1}\right|$, and other quantities.

Proof. We divide the proof into several lemmas. For the moment, we denote $E\left(u(t), u^{\prime}(t)\right)$ by $E(t)$.

Lemma 3.3. Let $u(t)$ be a local solution to the problem (1.1) on $[0, T[, T>0$. Then

$$
\forall 0 \leq S \leq T<+\infty, \quad E(S)-E(T)=\int_{S}^{T} \int_{\Omega} a(x) u^{\prime} g\left(u^{\prime}\right) d x d t .
$$

Multiplying the equation in (1.1) by $u^{\prime}(t)$ and integrating on $[S, T$, we get

$$
\begin{aligned}
-\int_{S}^{T} \int_{\Omega} a(x) u^{\prime} g\left(u^{\prime}\right) d x d t & =\left[\frac{1}{2}\left|u^{\prime}(t)\right|^{2}+\frac{1}{2} \widetilde{M}\left(|\nabla u(t)|^{2}\right)+F(u(t))\right]_{S}^{T} \\
& =E(T)-E(S) .
\end{aligned}
$$

It is easy to see the identity

$$
E^{\prime}(t)=-\int_{\Omega} a u^{\prime} g\left(u^{\prime}\right) d x \leq 0 .
$$


224 Equation with localized damping and source terms

In particular, $E(t)$ is nonincreasing and

$$
E(t) \leq E(0)
$$

as long as the local solutions exist.

Lemma 3.4. Let $u(t)$ be a local solution to the problem (1.1) satisfying $\left(u(t), u^{\prime}(t)\right) \in W_{K}$ on $[0, T[$ for some $K>0$. Then,

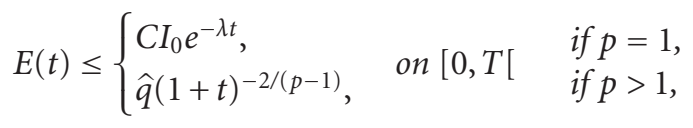

where $I_{0}^{2}=E(0), \lambda=\lambda\left(K, I_{0}\right)$, and $\hat{q}=\hat{q}\left(K, I_{0}\right)$ denote certain positive constants continuously depending on $K$ and $I_{0}$.

The proof of this lemma is based on the following identities given by the multiplier method. We omit to write the differential elements in the integrals, in order to simplify the expressions.

Lemma 3.5. Let $q \in\left[W^{1, \infty}(\Omega)\right]^{N}, \beta \in \mathbb{R}$, and $\xi \in W^{1, \infty}(\Omega)$. Then

$$
\begin{aligned}
\int_{S}^{T} \int_{\Gamma} M\left(|\nabla u(t)|^{2}\right) q \cdot v\left|\frac{\partial u}{\partial v}\right|^{2} E^{\sigma} \\
=\left.\left(u^{\prime}, 2 q \cdot \nabla u+\beta u\right) E^{\sigma}\right|_{S} ^{T}+\int_{S}^{T} \int_{\Omega}(\operatorname{div}(q)-\beta)\left[\left|u^{\prime}\right|^{2}-M\left(|\nabla u(t)|^{2}\right)|\nabla u(t)|^{2} E^{\sigma}\right] \\
\quad+2 \int_{S}^{T} \int_{\Omega} M\left(|\nabla u(t)|^{2}\right) \frac{\partial q_{k}}{\partial x_{i}} \frac{\partial u}{\partial x_{k}} \frac{\partial u}{\partial x_{i}} E^{\sigma}-\sigma \int_{S}^{T} \int_{\Omega} u^{\prime}(2 q \cdot \nabla u+\beta u) E^{\sigma-1} E^{\prime} \\
\quad+\int_{S}^{T} \int_{\Omega} a g\left(u^{\prime}\right)(2 q \cdot \nabla u+\beta u) E^{\sigma}-\int_{S}^{T} \int_{\Omega} \operatorname{div}(q) F(u) E^{\sigma}+\beta \int_{S}^{T} \int_{\Omega} f(u) \cdot u E^{\sigma},
\end{aligned}
$$

$$
\begin{aligned}
& \left.\left(u^{\prime}, \xi u\right) E^{\sigma}\right|_{S} ^{T}+\int_{S}^{T} \int_{\Omega} \xi\left[M\left(|\nabla u|^{2}\right)|\nabla u|^{2}-\left|u^{\prime}\right|^{2}\right] E^{\sigma} \\
& -\sigma \int_{S}^{T} \int_{\Omega} u^{\prime} u \xi E^{\sigma-1} E^{\prime}+\int_{S}^{T} \int_{\Omega} M\left(|\nabla u(t)|^{2}\right)(\nabla u, u \nabla \xi) E^{\sigma} \\
& +\int_{S}^{T} \int_{\Omega} a g\left(u^{\prime}\right) \xi u E^{\sigma}+\int_{S}^{T} \int_{\Omega} \xi f(u) u E^{\sigma}=0
\end{aligned}
$$

For the proof, see Lions [8] or Komornik [5]. 
Proof of Lemma 3.5. We proceed in several steps.

Step 1. Applying (3.9) with $q(x)=m(x)$, observing that $\operatorname{div} q=N$, we obtain

$$
\begin{aligned}
\left(u^{\prime}, 2 m\right. & \cdot \nabla u+\beta u)\left.E^{\sigma}\right|_{S} ^{T}+(N-\beta) \int_{S}^{T} \int_{\Omega}\left|u^{\prime}\right|^{2} E^{\sigma} \\
& +(\beta-N+2) \int_{S}^{T} \int_{\Omega} M\left(|\nabla u|^{2}\right)|\nabla u|^{2} E^{\sigma}-\sigma \int_{S}^{T} \int_{\Omega} u^{\prime}(2 m \cdot \nabla u+\beta u) E^{\sigma-1} E^{\prime} \\
& +\int_{S}^{T} \int_{\Omega} a g\left(u^{\prime}\right)(2 m \cdot \nabla u+\beta u) E^{\sigma}+\beta \int_{S}^{T} \int_{\Omega} f(u) \cdot u E^{\sigma}-N \int_{S}^{T} \int_{\Omega} F(u) E^{\sigma} \\
= & \int_{S}^{T} \int_{\Gamma} M\left(|\nabla u|^{2}\right)\left|\frac{\partial u}{\partial \nu}\right|^{2} E^{\sigma} \leq R M_{0} \int_{S}^{T} \int_{\Gamma_{0}}\left|\frac{\partial u}{\partial \nu}\right|^{2} E^{\sigma},
\end{aligned}
$$

where

$$
m_{0} \leq M\left(|\nabla u|^{2}\right) \leq \max \left\{M(s), 0 \leq s \leq \frac{4 E(0)}{m_{0}}\right\} \equiv M_{0}
$$

Throughout the remaining part of this work, positive constants will be denoted by $C$ and will change line to line. Here, we observe that under the assumption $\left(u(t), u^{\prime}(t)\right) \in$ $W_{K}$, the functionals $E(t), e(t)=(1 / 2)\left(\left|u^{\prime}(t)\right|^{2}+|\nabla u(t)|^{2}\right)$ and $\left|u^{\prime}(t)\right|^{2}+I(u(t))$ are all equivalent, by Lemma 2.4 .

We take $\beta \in] N-2, N\left[\right.$ and $\theta_{0}=\min \{2(N-\beta), \beta-N+2\}$, we deduce that

$$
\begin{aligned}
\theta_{0} \int_{S}^{T} E^{\sigma+1} \leq & R M_{0} \int_{S}^{T} \int_{\Gamma_{0}}\left|\frac{\partial u}{\partial v}\right|^{2} E^{\sigma}+\left|\left(u^{\prime}, 2 m \cdot \nabla u+\beta u\right)\right|_{S}^{T} \mid \\
& +\left|\int_{S}^{T} \int_{\Omega} a g\left(u^{\prime}\right)(2 m \cdot \nabla u+\beta u) E^{\sigma}\right| \\
& +\sigma\left|\int_{S}^{T} \int_{\Omega} u^{\prime}(2 m \cdot \nabla u+\beta u) E^{\sigma-1} E^{\prime}\right| \\
& +\beta\left|\int_{S}^{T} \int_{\Omega} f(u) \cdot u E^{\sigma}\right|+N\left|\int_{S}^{T} \int_{\Omega} F(u) E^{\sigma}\right| .
\end{aligned}
$$

Since the energy is nonincreasing, using the result of Komornik [5], we find that

$$
\begin{gathered}
\left|\left(u^{\prime}, 2 m \cdot \nabla u+\beta u\right)\right|_{S}^{T} E^{\sigma} \mid \leq C E(S), \\
\left|\sigma \int_{S}^{T} \int_{\Omega} u^{\prime}(2 m \cdot \nabla u+\beta u) E^{\sigma-1} E^{\prime}\right| \leq C E(S) .
\end{gathered}
$$


226 Equation with localized damping and source terms

By the Hölder inequality, we have

$$
\begin{aligned}
\left|\int_{S}^{T} \int_{\Omega_{1}} a g\left(u^{\prime}\right)(2 m \cdot \nabla u+\beta u) E^{\sigma}\right| & \leq \int_{S}^{T} E^{\sigma}\left(\int_{\Omega_{1}} a^{2} g^{2}\left(u^{\prime}\right)\right)^{1 / 2}\left(\int_{\Omega_{1}}|2 m \cdot \nabla u+\beta u|\right)^{1 / 2} \\
& \leq \int_{S}^{T} E^{\sigma}\left(\int_{\Omega_{1}} a^{2}\left(u^{\prime} g\left(u^{\prime}\right)\right)^{2 /(p+1)}\right)^{1 / 2} E^{1 / 2} \\
& \leq C \int_{S}^{T} E^{\sigma+1 / 2}\left|E^{\prime}\right|^{1 /(p+1)}, \\
\left|\int_{S}^{T} \int_{\Omega_{2}} a g\left(u^{\prime}\right)(2 m \cdot \nabla u+\beta u) E^{\sigma}\right| & \leq C \int_{S}^{T} E^{\sigma}\left\{\int_{\Omega_{2}}\left|a g\left(u^{\prime}\right)\right||\nabla u|+\int_{\Omega_{2}}\left|a g\left(u^{\prime}\right)\right||u|\right\} \\
& \leq C \int_{S}^{T} E^{\sigma}\left|a g\left(u^{\prime}\right)\right|_{1+q^{-1}}\left\{|\nabla u|_{q+1}+|\nabla u|\right\} .
\end{aligned}
$$

We observe here, from Lemma 2.2, that

$$
\begin{aligned}
|\nabla u|_{q+1} \leq C|\nabla u|^{1 /(q+1)}|\Delta u|^{q /(q+1)} \leq C K^{q /(q+1)} E^{1 / 2(q+1)}, & \\
\left|a g\left(u^{\prime}\right)\right|_{1+q^{-1}} & =\left[\int_{\Omega_{2}}\left|a g\left(u^{\prime}\right)\right|^{(q+1) / q}\right]^{q /(q+1)} \\
\leq & C\left[\int_{\Omega_{2}} a u^{\prime} g\left(u^{\prime}\right)\right]^{q /(q+1)} \leq C\left|E^{\prime}\right|^{q /(q+1)} .
\end{aligned}
$$

From (3.17), (3.18), and (3.19), we have

$$
\left|\int_{S}^{T} \int_{\Omega_{2}} a g\left(u^{\prime}\right)(2 m \cdot \nabla u+\beta u) E^{\sigma}\right| \leq C \int_{S}^{T}|E|^{\sigma+1 / 2(q+1)}\left|E^{\prime}\right|^{q /(q+1)},
$$

where we set for each $t \geq 0$,

$$
\Omega_{1}=\Omega_{1}(t)=\left\{x \in \Omega:\left|u^{\prime}(x, t)\right| \leq 1\right\}, \quad \Omega_{2}=\Omega \backslash \Omega_{1} .
$$

Thus, from (3.16) and (3.20), we get

$$
\left|\int_{S}^{T} \int_{\Omega} a g\left(u^{\prime}\right)(2 m \cdot \nabla u+\beta u) E^{\sigma}\right| \leq C \int_{S}^{T}\left[E^{\sigma+1 / 2}\left|E^{\prime}\right|^{1 /(p+1)}+|E|^{\sigma+1 / 2(q+1)}\left|E^{\prime}\right|^{q /(q+1)}\right] .
$$

Now, using the Young inequality, we obtain

$$
\left|\int_{S}^{T} \int_{\Omega} a g\left(u^{\prime}\right)(2 m \cdot \nabla u+\beta u) E^{\sigma}\right| \leq \varepsilon \int_{S}^{T} E^{\sigma+1}+E(S), \quad \varepsilon>0 .
$$


It follows from (3.14), (3.15), and (3.23) that

$$
\frac{\theta_{0}}{2} \int_{S}^{T} E^{\sigma+1} \leq C E(S)+\int_{S}^{T} \int_{\Omega}\left(\frac{\beta^{2}}{\theta_{0}}|f(u)|^{2}+N F(u)\right) E^{\sigma}+R M_{0} \int_{S}^{T} \int_{\Gamma_{0}}\left|\frac{\partial u}{\partial v}\right|^{2} E^{\sigma} .
$$

To estimate the last term in (3.24), we utilize (3.10) with $\xi=\eta$, where $\eta \in W^{1, \infty}(\Omega)$ is a function (constructed by Zuazua in [10]) which satisfies

$$
\begin{gathered}
0 \leq \eta \leq 1, \\
\eta=1, \quad \text { in } \widehat{\omega}, \quad \frac{|\nabla \eta|^{2}}{\eta} \in L^{\infty}(\omega), \\
\eta=0, \quad \text { in } \Omega \backslash \omega,
\end{gathered}
$$

and $\hat{\omega}$ is an open set in $\Omega$, with $\Gamma_{0} \subseteq \widehat{\omega} \varsubsetneqq \omega$.

First, we have from (3.10)

$$
\begin{aligned}
\int_{S}^{T} \int_{\Omega} \eta M\left(|\nabla u|^{2}\right)|\nabla u|^{2} E^{\sigma}= & \left.\left(-u^{\prime}, \eta u\right) E^{\sigma}\right|_{S} ^{T}-\int_{S}^{T} \int_{\Omega} a g\left(u^{\prime}\right) u \eta E^{\sigma} \\
& -\int_{S}^{T} \int_{\Omega} M\left(|\nabla u|^{2}\right) \nabla u \cdot u \nabla \eta E^{\sigma} \\
& +\int_{S}^{T} \int_{\Omega} \eta\left|u^{\prime}\right|^{2} E^{\sigma}-\int_{S}^{T} \int_{\Omega} \eta f(u) u E^{\sigma} \\
& +\sigma \int_{S}^{T} \int_{\Omega} u^{\prime} u \xi E^{\sigma-1} E^{\prime}
\end{aligned}
$$

Simple calculations, using the Young inequalities, show that

$$
\begin{gathered}
\left|-\left(u^{\prime}, \eta u\right) E^{\sigma}\right|_{S}^{T}+\sigma \int_{S}^{T} \int_{\Omega} u^{\prime} u \xi E^{\sigma-1} E^{\prime} \mid \leq C E(S), \\
\left|\int_{S}^{T}\left(a g\left(u^{\prime}\right), \eta u\right) E^{\sigma}\right| \leq C E(S)+\frac{\varepsilon}{2} \int_{S}^{T} E^{\sigma+1}, \quad \varepsilon>0, \\
\left|-\int_{S}^{T} \int_{\Omega} M\left(|\nabla u|^{2}\right) \nabla u \cdot u \nabla \eta E^{\sigma}\right| \leq C \int_{S}^{T} \int_{\omega}|u|^{2} E^{\sigma}+\frac{1}{2} \int_{S}^{T} \int_{\Omega} \eta M\left(|\nabla u|^{2}\right)|\nabla u|^{2} E^{\sigma} .
\end{gathered}
$$

From (3.26)-(3.29), we obtain

$$
\begin{aligned}
\frac{1}{2} \int_{S}^{T} \int_{\Omega} \eta M\left(|\nabla u|^{2}\right)|\nabla u|^{2} E^{\sigma} \leq & C\left[E(S)+\int_{S}^{T} \int_{\omega}\left(\left|u^{\prime}\right|^{2}+|u|^{2} E^{\sigma}\right)\right] \\
& +\frac{C_{*}}{m_{0}^{2}} \int_{S}^{T} \int_{\Omega}|f(u)|^{2} E^{\sigma}+\varepsilon \int_{S}^{T} E^{\sigma+1} .
\end{aligned}
$$

Step 2. We take a vector field $h \in\left[W^{1, \infty}(\Omega)\right]^{N}$ such that

$$
h=v, \quad \text { on } \Gamma_{0}, \quad h \cdot v \geq 0, \quad \text { on } \Gamma, \quad h=0, \quad \text { on } \Omega \backslash \hat{\omega} .
$$


228 Equation with localized damping and source terms

Choosing $\beta=0$ and $q=h$ in (3.9), we get

$$
\begin{aligned}
&\left|\int_{S}^{T} \int_{\Omega} a g\left(u^{\prime}\right) h \cdot\right| \nabla u\left|E^{\sigma}\right| \leq C \int_{S}^{T}\left[E^{\sigma+1 / 2}\left|E^{\prime}\right|^{1 /(p+1)}+E^{\sigma+1 / 2(q+1)}\left|E^{\prime}\right|^{q /(q+1)}\right], \\
& m_{0} \int_{S}^{T} \int_{\Gamma_{0}}\left|\frac{\partial u}{\partial \nu}\right|^{2} E^{\sigma} \leq C E(S)+3 \alpha_{0} \int_{S}^{T} \int_{\widehat{\omega}}\left(\left|u^{\prime}\right|^{2}+M\left(|\nabla u|^{2}\right)|\nabla u|^{2}\right) E^{\sigma} \\
&+\alpha_{0} \int_{S}^{T} \int_{\Omega}|F(u)| E^{\sigma}+\varepsilon^{\prime} \int_{S}^{T} E^{\sigma+1}, \quad \varepsilon^{\prime}>0
\end{aligned}
$$

where $\sum_{i, j=1}^{N}\left|\partial h_{j} / \partial x_{i}\right| \leq \alpha_{0}$, for all $x \in \bar{\Omega}$.

Combining (3.30) and (3.32), we have

$$
\begin{aligned}
m_{0} \int_{S}^{T} \int_{\Gamma_{0}}\left|\frac{\partial u}{\partial v}\right|^{2} E^{\sigma} \leq & C\left[E(S)+\int_{S}^{T} \int_{\omega}\left(\left|u^{\prime}\right|^{2}+|u|^{2}\right) E^{\sigma}\right] \\
& +\frac{\sigma C_{*}^{2} \alpha_{0}}{m_{0}^{2}} \int_{S}^{T} \int_{\Omega}|f(u)|^{2} E^{\sigma}+\alpha_{0} \int_{S}^{T} \int_{\Omega}|F(u)| E^{\sigma}+\varepsilon \int_{S}^{T} E^{\sigma+1}
\end{aligned}
$$

We conclude from (3.24) and (3.33) that

$$
\begin{aligned}
\frac{\theta_{0}}{2} \int_{S}^{T} E^{\sigma+1} \leq & \left(\frac{\sigma C_{*}^{2} \alpha_{0} R M_{0}}{m_{0}^{3}}+\frac{\beta^{2}}{\theta_{0}}\right) \int_{S}^{T} \int_{\Omega}|f(u)|^{2} \\
& +\left(N+\frac{\alpha_{0} R M_{0}}{m_{0}}\right) \int_{S}^{T} \int_{\Omega}|F(u)| \\
& +C\left[E(S)+\int_{S}^{T} \int_{\omega}\left(\left|u^{\prime}\right|^{2}+|u|^{2}\right)\right] .
\end{aligned}
$$

Now, in order to absorb the last term into the right-hand side of (3.34), we adapt a method introduced in Conrad and Rao [4]. To this end, we consider $z(t) \in H_{0}^{1}(\Omega)$, solution of

$$
-\Delta z=\chi(\omega) u, \quad \text { in } \Omega, \quad z=0, \quad \text { on } \Gamma,
$$

where $\chi(\omega)$ is the characteristic function of $\omega$. It is easy to verify that $z^{\prime}$ is solution of the problem

$$
-\Delta z^{\prime}=\chi(\omega) u^{\prime}, \quad \text { in } \Omega, \quad z^{\prime}=0, \quad \text { on } \Gamma .
$$

A simple computation gives

$$
|z| \leq C|u|_{L^{2}(\omega)}, \quad\left|z^{\prime}\right| \leq C\left|u^{\prime}\right|_{L^{2}(\omega)}, \quad(\nabla z, \nabla u)=|u|_{L^{2}(\omega)}^{2}
$$


Next, we "multiply" the equation in (1.1) by $z E^{\sigma}$, integrate by parts on $\left.\Omega \times\right] S, T$, and use (3.37). Thus, we find

$$
\begin{aligned}
\int_{S}^{T} \int_{\omega} M\left(|\nabla u|^{2}\right)|\nabla u|^{2} E^{\sigma}= & -\left.\left(u^{\prime}, z\right) E^{\sigma}\right|_{S} ^{T}+\int_{S}^{T}\left(u^{\prime}, z^{\prime}\right) E^{\sigma}+\sigma \int_{S}^{T} E^{\sigma-1} E^{\prime}\left(u^{\prime}, z^{\prime}\right) \\
& -\int_{S}^{T}\left(a g\left(u^{\prime}\right), z\right) E^{\sigma}-\int_{S}^{T}(f(u), z) E^{\sigma} .
\end{aligned}
$$

Here, we note that

$$
\begin{gathered}
\left|-\left(u^{\prime}, z\right) E^{\sigma}\right|_{S}^{T}+\sigma \int_{S}^{T} E^{\sigma-1} E^{\prime}\left(u^{\prime}, z^{\prime}\right) \mid \leq C E(S) \\
\left|\int_{S}^{T}\left(u^{\prime}, z\right) E^{\sigma}\right| \leq C \int_{S}^{T} \int_{\omega}\left|u^{\prime}\right|^{2} E^{\sigma}+\varepsilon \int_{S}^{T} E^{\sigma+1}, \quad \varepsilon>0, \\
\left|\int_{S}^{T}\left(a g\left(u^{\prime}\right), z\right) E^{\sigma}\right| \leq C E(S)+\varepsilon^{\prime} \int_{S}^{T} E^{\sigma+1}, \quad \varepsilon^{\prime}>0, \\
\left|-\int_{S}^{T}(f(u), z) E^{\sigma}\right| \leq \frac{1}{2 m_{0}} \int_{S}^{T} \int_{\Omega}|f(u)|^{2} E^{\sigma}+\frac{m_{0}}{2} \int_{S}^{T} \int_{\omega}|u|^{2} E^{\sigma} .
\end{gathered}
$$

Using (3.39)-(3.41), we have in (3.38)

$$
\int_{S}^{T} \int_{\omega}|u|^{2} E^{\sigma} \leq C\left(E(S)+\int_{S}^{T} \int_{\omega}\left|u^{\prime}\right|^{2} E^{\sigma}\right)+\frac{1}{m_{0}^{2}} \int_{S}^{T} \int_{\Omega}|f(u)|^{2} E^{\sigma}+\varepsilon \int_{S}^{T} E^{\sigma+1}, \quad \varepsilon>0 .
$$

Then inserting (3.43) into (3.34) gives

$$
\int_{S}^{T} E^{\sigma+1} \leq C\left(E(S)+\int_{S}^{T} \int_{\omega}\left|u^{\prime}\right|^{2} E^{\sigma}\right)+\delta_{0} \int_{S}^{T} \int_{\Omega}|f(u)|^{2} E^{\sigma}+\delta_{1} \int_{S}^{T}|F(u)| E^{\sigma},
$$

where

$$
\delta_{0}=\frac{8}{\theta_{0}}\left[\frac{\sigma C_{*} \alpha_{0} R M_{0}}{m_{0}^{3}}+\frac{\beta^{2}}{\theta_{0}}+\frac{1}{m_{0}^{2}}\right], \quad \delta_{1}=\frac{8}{\theta_{0}}\left[N+\frac{\alpha_{0} R M_{0}}{m_{0}}\right] .
$$

Now, we observe that

$$
\begin{aligned}
\delta_{0} \int_{\Omega}|f(u)|^{2} & \leq \delta_{0} h_{0}|u|_{2(\alpha+1)}^{2(\alpha+1)} \leq C \delta_{0} h_{0}|u|_{2 N /(N-2)}^{2\left(1-\theta_{1}\right)(\alpha+1)}|\Delta u|^{2 \theta_{1}(\alpha+1)} \\
& \leq C \delta_{0} h_{0} \varepsilon_{0}^{2\left(1-\theta_{1}\right)(\alpha+1)-2} K^{2 \theta_{1}(\alpha+1)}|\nabla u|^{2}
\end{aligned}
$$

if $N \geq 3$ and $\alpha>2 /(N-2)$, with $\theta_{1}=((\alpha+1)(N-2)-N) / 2(\alpha+1)$.

Further, we have that since $\alpha \leq 2 /(N-4)^{+}$,

$$
\left(1-\theta_{1}\right)(\alpha+1) \geq 1 \text {. }
$$

Thus,

$$
\delta_{0} h_{0}|u|_{2(\alpha+1)}^{2(\alpha+1)} \leq C \delta_{0} h_{0} \varepsilon_{0}^{2\left(1-\theta_{1}\right)(\alpha+1)-2} K^{2 \theta_{1}(\alpha+1)}|\nabla u|^{2} .
$$


When $N \leq 2$ or $\alpha \leq 2 /(N-2)^{+}$, we see that (3.48) holds with $\theta_{1}=0$. Thus, under a little more stronger assumption than (2.19),

$$
C h_{0}\left(\varepsilon_{0}^{(\alpha+2)\left(1-\theta_{1}\right)-2} K^{(\alpha+2) \theta}+\varepsilon_{0}^{2\left(1-\theta_{1}\right)(\alpha+1)-2} K^{2 \theta_{1}(\alpha+1)}\right) \leq \frac{m_{0}}{4},
$$

we obtain from (3.44) that

$$
\int_{S}^{T} E^{\sigma+1} d t \leq C\left(E(S)+\int_{S}^{T} \int_{\omega}\left|u^{\prime}\right|^{2} E^{\sigma}\right)
$$

In order to absorb the second term in (3.50), we consider two cases.

(i) The case $p=1$. We take $\sigma=0$, hence by (2.6),

$$
\int_{S}^{T} E \leq C E(S)+\frac{C}{a_{0}} \int_{S}^{T} \int_{\omega} a\left|u^{\prime}\right|^{2} \leq C E(S)+\int_{S}^{T} \int_{\omega} a\left|g\left(u^{\prime}\right)\right| u^{\prime} \leq C E(S) .
$$

Applying Lemma 2.3, we obtain

$$
E(t) \leq C I_{0} e^{-\lambda t}, \quad \lambda=\lambda\left(K, I_{0}\right) .
$$

(ii) When $p>1$, we take $\sigma=(p-1) / 2$. It follows from (2.6) that

$$
\begin{aligned}
\int_{S}^{T} E^{(p+1) / 2} & \leq C E(S)+\frac{C}{a_{0}} \int_{S}^{T} \int_{\omega} a\left|u^{\prime}\right|^{2} E^{(p-1) / 2} \\
& \leq C E(S)+C \int_{S}^{T} \int_{\Omega} a\left|u^{\prime}\right|^{2} E^{(p-1) / 2} \\
& \leq C\left[E(S)+\int_{S}^{T} E^{(p-1) / 2}\left(\int_{\Omega_{1}} a\left|u^{\prime}\right|^{2}+\int_{\Omega_{2}} a\left|u^{\prime}\right|^{2}\right)\right] \\
& \leq C\left[E(S)+\int_{S}^{T}\left(E^{(p-1) / 2}\left|E^{\prime}\right|^{2 /(p+1)}+E^{(p-1) / 2}\left|E^{\prime}\right|\right)\right] \\
& \leq C\left[E(S)+E^{(p+1) / 2}(S)\right]+\varepsilon \int_{S}^{T} E^{(p+1) / 2}, \quad \varepsilon>0 .
\end{aligned}
$$

Hence

$$
\int_{S}^{T} E^{(p+1) / 2} \leq C E(S)
$$

Using Lemma 2.3 again, we conclude that

$$
E(t) \leq q\left(K, I_{0}\right)(1+t)^{-2 /(p-1)}, \quad t \geq 0,
$$

as long as the local solutions exist, where $q\left(K, I_{0}\right)$ denotes a certain constant continuously depending on $K$ and $I_{0}$.

We are now in a position to obtain $H^{2}$ a priori bounds. Set

$$
E_{1}(t)=M\left(|\nabla u(t)|^{2}\right)|\Delta u(t)|^{2}+\left|\nabla u^{\prime}(t)\right|^{2} .
$$

Then we have the following estimate, which is the heart of this paper. 
Lemma 3.6. Assume that $u(t)$ is a solution satisfying $\left(u(t), u^{\prime}(t)\right) \in W_{K}$ on $[0, T[$, for some $K>0$. Then,

$$
\left|\nabla u^{\prime}(t)\right|^{2}+|\Delta u(t)|^{2} \leq Q^{2}\left(I_{0}, I_{1}, K\right)
$$

with

$$
\lim _{I_{0} \rightarrow 0} Q^{2}\left(I_{0}, I_{1}, K\right)=\frac{I_{1}^{2}}{\min \left\{1, m_{0}\right\}},
$$

and where

$$
I_{1}^{2}=\left|\nabla u_{1}\right|^{2}+M\left(\left|\nabla u_{0}\right|^{2}\right)\left|\Delta u_{0}\right|^{2}
$$

Proof. For $E_{1}(t)$ with respect to $t$, we get

$$
\begin{aligned}
& \frac{1}{2} \frac{d}{d t} E_{1}(t)+\left(a g^{\prime}\left(u^{\prime}\right) \nabla u^{\prime}(t), \nabla u^{\prime}(t)\right) \\
& \quad=-\left(f^{\prime}(u) \nabla u, \nabla u^{\prime}\right)+M^{\prime}\left(|\nabla u(t)|^{2}\right)\left(\nabla u^{\prime}(t), \nabla u(t)\right)|\Delta u(t)|^{2}-\left(g\left(u^{\prime}\right) \nabla a, \nabla u^{\prime}\right) .
\end{aligned}
$$

Using the assumptions on $a, g$, and $f$, it follows from (3.60) that

$$
\begin{aligned}
\frac{d}{d t} E_{1}(t) & \leq C\left\{\int_{\Omega}\left(|u|^{\alpha}|\nabla u|\left|\nabla u^{\prime}\right|\right)+|\nabla u|_{2}^{\gamma+1}\left|\nabla u^{\prime}\right|_{2}|\Delta u|_{2}^{2}+\sum_{i=1}^{n} \int_{\Omega} \frac{\partial a}{\partial x_{i}} \frac{\partial}{\partial x_{i}} \int_{0}^{u^{\prime}} g(\xi) d \xi\right\} \\
& \leq C\left\{\int_{\Omega}\left(|u|^{\alpha}|\nabla u|\left|\nabla u^{\prime}\right|\right)+|\nabla u|_{2}^{\gamma+1}\left|\nabla u^{\prime}\right|_{2}|\Delta u|_{2}^{2}+\int_{\Omega}\left|\int_{0}^{u^{\prime}} g\right||\Delta a|\right\} \\
& \leq C\left\{\left(\int_{\Omega}|u|^{2 \alpha}|\nabla u|^{2}\right)^{1 / 2}\left(\int_{\Omega}\left|\nabla u^{\prime}\right|^{2}\right)^{1 / 2}+E(t)^{(\gamma+1) / 2} K^{3}+\int_{\Omega} a g\left(u^{\prime}\right) u^{\prime}\right\} .
\end{aligned}
$$

Further, we observe that

$$
\begin{aligned}
\left(\int_{\Omega}|u|^{2 \alpha}|\nabla u|^{2}\right)^{1 / 2} & \leq|u|_{N \alpha}^{\alpha}|\nabla u|_{2 N /(N-2)} \\
& \leq C|u|_{2 N /(N-2)}^{\alpha\left(1-\theta_{0}\right)}|\Delta u|_{2}^{\alpha \theta_{0}}|\Delta u|_{2} \quad \text { (by Lemma 2.2) } \\
& \leq C|\nabla u|_{2}^{\alpha\left(1-\theta_{0}\right)}|\Delta u|_{2}^{\alpha \theta_{0}+1} \\
& \leq C E(t)^{\alpha\left(1-\theta_{0}\right) / 2} K^{\alpha \theta_{0}+1},
\end{aligned}
$$

with

$$
\theta_{0}=\left(\frac{N-2}{2}-\frac{1}{\alpha}\right)^{+}=\frac{((N-2) \alpha-2)^{+}}{2 \alpha} \leq 1 .
$$

Then, it follows from (3.61) and (3.62) that

$$
\frac{d}{d t} E_{1}(t) \leq C\left\{E(t)^{\alpha\left(1-\theta_{0}\right) / 2} K^{\alpha \theta_{0}+2}+E(t)^{(\gamma+1) / 2} K^{3}-E^{\prime}(t)\right\} .
$$


232 Equation with localized damping and source terms

Thus, integrating (3.64) over $\left[0, t\left[\right.\right.$, we obtain $\int_{\Omega}\left|\int_{0}^{u^{\prime}} g\right||\Delta a|$,

$$
\begin{aligned}
& |\Delta u(t)|^{2}+\left|\nabla u^{\prime}(t)\right|^{2} \\
& \quad \leq \frac{1}{\min \left\{1, m_{0}\right\}}\left\{I_{1}^{2}+C K^{\alpha \theta_{0}+2} \int_{0}^{+\infty} E(t)^{\alpha\left(1-\theta_{0}\right) / 2} d t+C K^{3} \int_{0}^{+\infty} E(t)^{(\gamma+1) / 2} d t+C I_{0}^{2}\right\} \\
& \quad \leq \frac{1}{\min \left\{1, m_{0}\right\}}\left\{I_{1}^{2}+C I_{0}^{2}+C I_{0}^{(\gamma+1) / 2} K^{3}+C I_{0}^{\alpha\left(1-\theta_{0}\right) / 2} K^{\alpha \theta_{0}+2}\right\} \\
& \quad \equiv Q^{2}\left(I_{0}, I_{1}, K\right), \quad \text { on }[0, T[.
\end{aligned}
$$

Define

$$
\begin{gathered}
S_{K}=\left\{\left(u_{0}, u_{1}\right) \in\left(H_{0}^{1} \cap H^{2}\right) \times H_{0}^{1}: Q^{2}\left(I_{0}, I_{1}, K\right)<K\right\}, \\
S_{0}=\bigcup_{K>0} S_{K} .
\end{gathered}
$$

Since

$$
\lim _{I_{0} \rightarrow 0} Q^{2}\left(I_{0}, I_{1}, K\right)=\frac{I_{1}^{2}}{\min \left\{1, m_{0}\right\}},
$$

the $S_{K}$ is not empty if $I_{1}<\min \left\{1, m_{0}\right\} K^{2}$ and $I_{0}$ is sufficiently small.

If $\left(u_{0}, u_{1}\right) \in S_{K}$ for some $K>0$, then the corresponding local solution $u(t)$ exists on some interval $[0, T[$ and satisfies

$$
E_{1}(u(t)) \leq \min \left\{1, m_{0}\right\} K^{2} \quad \text { on } 0 \leq t<T .
$$

From (3.43), we have $\left(u(t), u^{\prime}(t)\right) \in W_{K}$.

Hence, it follows from Lemma 3.6 that

$$
\left|\nabla u^{\prime}(t)\right|^{2}+|\Delta u(t)|^{2} \leq Q^{2}\left(I_{0}, I_{1}, K\right)<K^{2}, \quad \text { on }[0, T[.
$$

Next, we affirm that

$$
\left(u(t), u^{\prime}(t)\right) \in S_{K}, \quad \text { on }[0, T[.
$$

In fact, suppose that there is a number $t^{*} \in\left[0, T\left[\right.\right.$ such that $\left(u(t), u^{\prime}(t)\right) \in S_{K}$ on $[0, T[$ and $\left(u\left(t^{*}\right), u^{\prime}\left(t^{*}\right)\right) \notin S_{K}$, then it follows that

$$
Q^{2}\left(E\left(u\left(t^{*}\right)\right), E_{1}\left(u\left(t^{*}\right)\right), K\right) \geq K^{2} .
$$

Note that $Q^{2}\left(I_{0}, I_{1}, K\right)$ is the increasing function with respect to $I_{0}$ and $I_{1}$. Hence, we see from (3.48), the energy identity (3.4), and Lemma 3.6 that

$$
I_{1}^{2}+C I_{0}^{2}+C_{1}\left(I_{0}\right) K^{3}+C_{2}\left(I_{0}\right) K^{\alpha \theta_{0}+2} \geq \min \left\{1, m_{0}\right\} K^{2} .
$$

But, then, since

$$
\lim _{I_{0} \rightarrow 0} Q^{2}\left(I_{0}, I_{1}, K\right)=\frac{I_{1}^{2}}{\min \left\{1, m_{0}\right\}},
$$


we may take $I_{1}$ so that

$$
\frac{2 I_{1}^{2}}{\min \left\{1, m_{0}\right\}}<K^{2}
$$

with sufficiently small $I_{0}$. This contradicts (3.72).

Now, since we can repeat the continuation procedure indefinitely, we conclude that $u(t)$ can be continued globally on $\left[0,+\infty\left[\right.\right.$ and $\left(u(t), u^{\prime}(t)\right) \in S_{0}$, for all $t \geq 0$.

The uniqueness follows from a similar argument as in [1].

\section{References}

[1] M. Aassila, Global existence of solutions to a wave equation with damping and source terms, Differential Integral Equations 14 (2001), no. 11, 1301-1314.

[2] E. Cabanillas, J. Bernui, and Z. Huaringa, Existencia Global y Decaimiento, de la Energía de una Ecuación de Kirchhoff con Disipación Localizada, Pesquimat Rev de la F.C.M 6 (2003), no. 1, $21-31$.

[3] _ Global Existence and Decay Estimates to a quasilinear wave equation with locally distributed Damping, Pesquimat, Rev de la F.C.M - UNMSM V N 2 (2003).

[4] F. Conrad and B. Rao, Decay of solutions of the wave equation in a star-shaped domain with nonlinear boundary feedback, Asymptotic Anal. 7 (1993), no. 3, 159-177.

[5] V. Komornik, Exact Controllability and Stabilization, the Multiplier Method, RAM: Research in Applied Mathematics, Masson, Paris, 1994.

[6] S. Kouémou-Patcheu, Global existence and exponential decay estimates for a damped quasilinear equation, Comm. Partial Differential Equations 22 (1997), no. 11-12, 2007-2024.

[7] J.-L. Lions, Quelques méthodes de résolution des problèmes aux limites non linéaires, Dunod, Paris, 1969.

[8] Contrôlabilité Exacte, Perturbations et Stabilisation de Systèmes Distribués. Tome 1. Contrôlabilité Exacte. [Exact Controllability, Perturbations and Stabilization of Distributed Systems. Vol. 1. Exact Controllability], Recherches en Mathématiques Appliquées [Research in Applied Mathematics], vol. 8, Masson, Paris, 1988.

[9] M. Nakao, Decay of solutions of the wave equation with a local degenerate dissipation, Israel J. Math. 95 (1996), 25-42.

[10] E. Zuazua, Exponential decay for the semilinear wave equation with locally distributed damping, Comm. Partial Differential Equations 15 (1990), no. 2, 205-235.

E. Cabanillas Lapa: Instituto de Investigación, Facultad de Ciencias Matemáticas, Universidad Nacional Mayor de San Marcos, Lima, Peru

E-mail address: cleugenio@yahoo.com

Z. Huaringa Segura: Instituto de Investigación, Facultad de Ciencias Matemáticas, Universidad Nacional Mayor de San Marcos, Lima, Peru

E-mail address: zachariashs@yahoo.es

F. Leon Barboza: Instituto de Investigación, Facultad de Ciencias Matemáticas, Universidad Nacional Mayor de San Marcos, Lima, Peru

E-mail address: fleonb2004@yahoo.es 


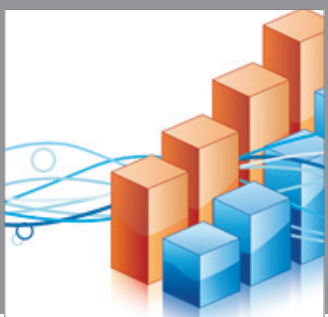

Advances in

Operations Research

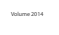

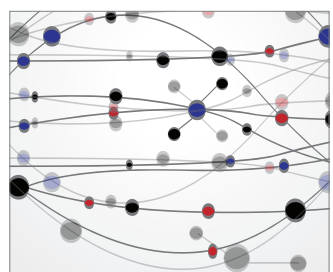

\section{The Scientific} World Journal
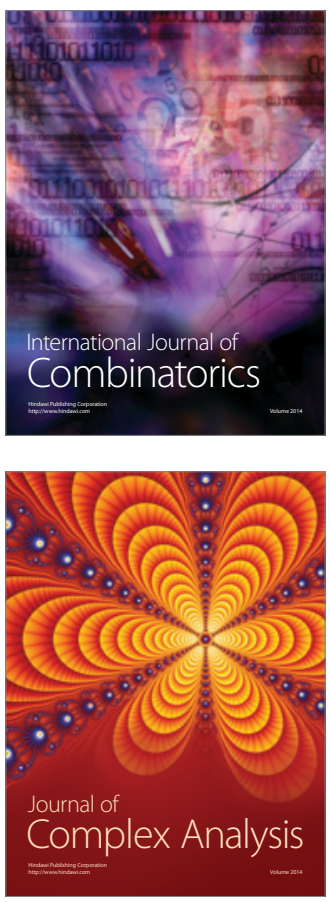

International Journal of

Mathematics and

Mathematical

Sciences
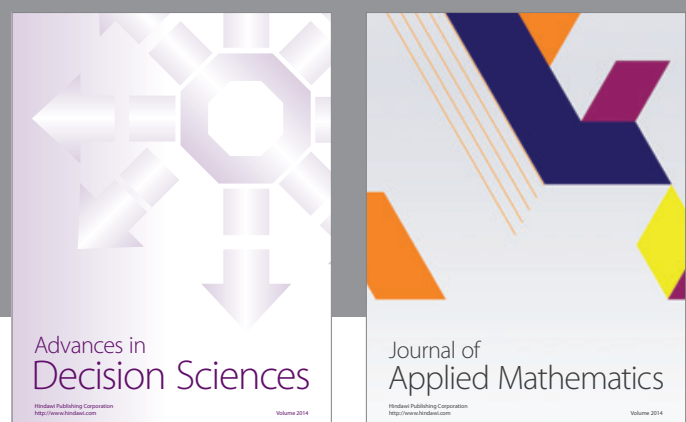

Journal of

Applied Mathematics
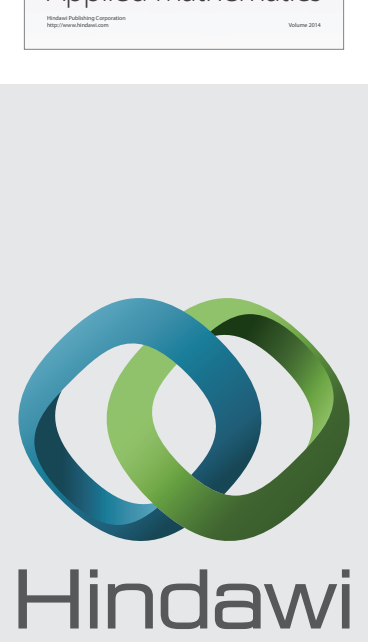

Submit your manuscripts at http://www.hindawi.com
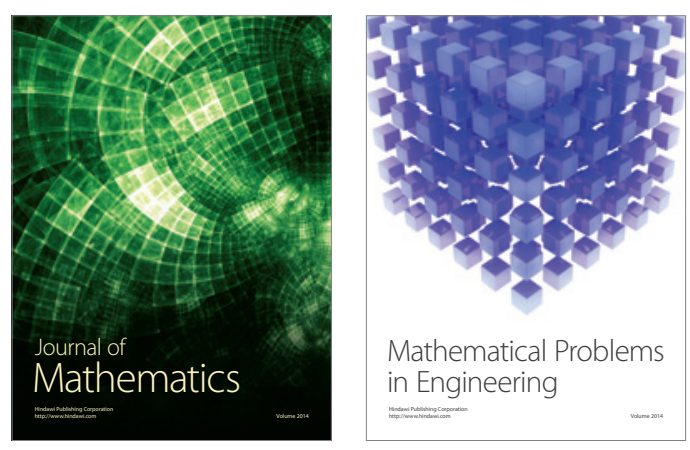

Mathematical Problems in Engineering
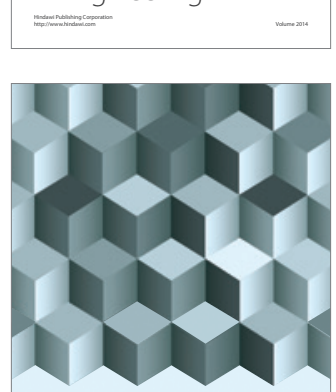

Journal of

Function Spaces
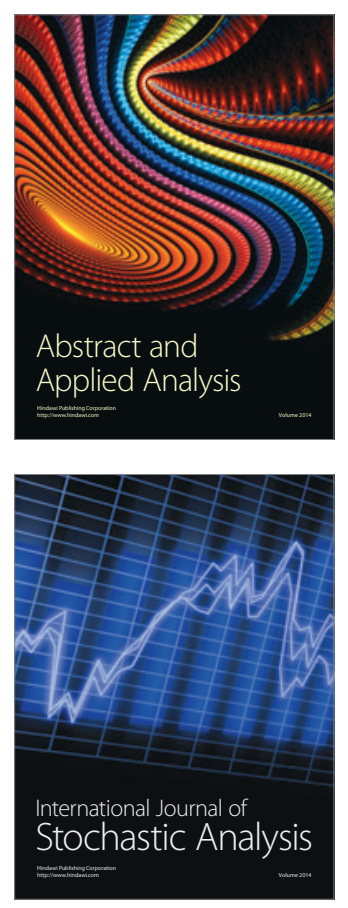

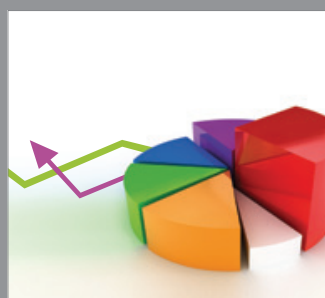

ournal of

Probability and Statistics

Promensencen
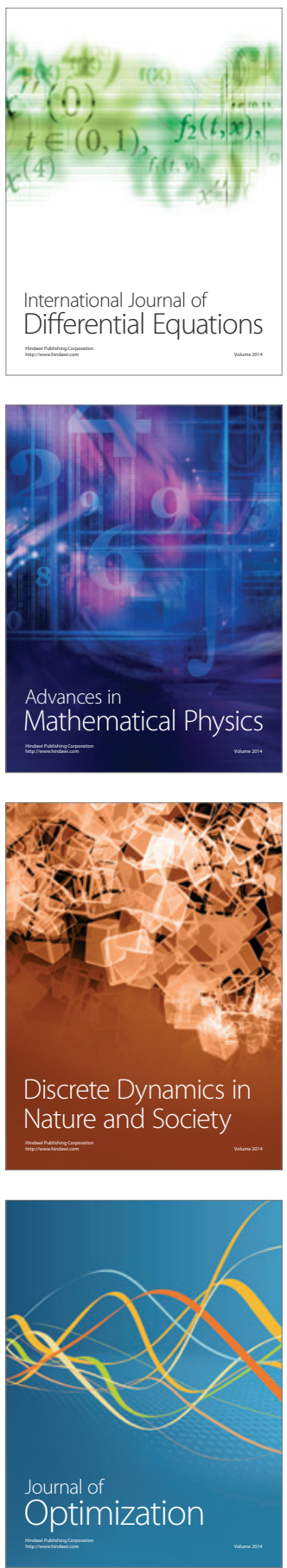